\title{
Time-Domain Green's Function-Based Parametric Sensitivity Analysis of Multiconductor Transmission Lines
}

\author{
Domenico Spina, Francesco Ferranti, Member, IEEE, Giulio Antonini, Senior Member, IEEE, \\ Tom Dhaene, Senior Member, IEEE, Luc Knockaert, Senior Member, IEEE, Dries Vande Ginste, Member, IEEE,
}

\begin{abstract}
We present a new parametric macromodeling technique for lossy and dispersive multiconductor transmission lines (MTLs). This technique can handle multiple design parameters, such as substrate or geometrical layout features, and provides time-domain sensitivity information for voltages and currents at the ports of the lines. It is derived from the dyadic Green's function of the 1-D wave propagation problem. The rational nature of the Green's function permits the generation of a timedomain macromodel for the computation of transient voltage and current sensitivities with respect to both electrical and physical parameters, completely avoiding similarity transformation and it is suited to generate state-space models and synthesize equivalent circuits, which can be easily embedded into conventional SPICElike solvers. Parametric macromodels which provide sensitivity information are well suited for design space exploration, design optimization and crosstalk analysis. Two numerical examples validates the proposed approach in both frequency and time domain.
\end{abstract}

Index Terms-Interconnects, parametric macromodeling, rational approximation, sensitivity analysis.

\section{INTRODUCTION}

The increasing demand for performance of integrated circuits (ICs) pushes operation to higher signal bandwidths, while rapid advances in manufacturing capabilities have significantly reduced the feature size and increased the density of these devices. To assist microwave designers, accurate modeling of previously neglected second order effects, such as crosstalk, reflection, delay and coupling, becomes increasingly important during circuit and system simulations. The accurate prediction of these interconnect effects is fundamental for a successful design and involves the solution of large systems of equations which are often prohibitively CPU expensive to solve [1], [2]. Furthermore, microwave designers have to make the proper trade-offs between conflicting design requirements using optimization techniques, to obtain the best possible performance considering physical effects such as reflection, crosstalk and

Domenico Spina, Francesco Ferranti, Tom Dhaene and Luc Knockaert are with the Department of Information Technology, Internet Based Communication Networks and Services (IBCN), Ghent University - IBBT, Gaston Crommenlaan 8 Bus 201, B-9050 Gent, Belgium, email: \{domenico.spina, francesco.ferranti, tom.dhaene, luc.knockaert $\} @$ intec.ugent.be;

Dries Vande Ginste is with the Department of of Information Technology, Electromagnetics Group, Ghent University, Sint-Pietersnieuwstraat 41, B-9000 Gent, Belgium, email: dries.vandeginste@intec.UGent.be;

Giulio Antonini is with the UAq EMC Laboratory, Dipartimento di Ingegneria Elettrica e dell'Informazione, Università degli Studi dell'Aquila, Via G. Gronchi 18, 67100, L'Aquila, Italy, phone: +390862434462, email: antonini@ing.univaq.it. propagation delays. For example, once the fabrication technology is decided, an optimization step is required at the early design stages to select the geometrical and material features of the structure, such as length and width of conductors, dielectric permittivity and metal conductivity, yielding the optimum electrical performance, often under stringent signal integrity and electromagnetic compatibility constraints. To perform these design activities using full electromagnetic simulations on the entire parameter space is often computationally expensive, therefore parametric macromodeling techniques that take into account design parameters in addition to frequency (or time) are needed [3], [4].

Recently, a spectral approach has been presented for the analysis of lossy and dispersive MTLs [5] and in [6] it is extended to provide a closed-form sensitivity analysis for MTLs in the frequency-domain. It is based on the computation of the closed-form dyadic Green's function of the 1-D wave propagation problem. The major advantage of such an approach over existing techniques [7], [8] is the rational nature of the dyadic Green's function which is appropriate for time-domain macromodeling and is suitable to generate a finite statespace representation and an equivalent SPICE circuit by using standard realization [9] and circuit synthesis techniques [10]. In [3], [4] a parametric macromodeling technique for lossy and dispersive MTLs is proposed, based on the spectral approach [5]. It provides time-domain information for voltage and current at the ports of the lines, starting from the knowledge of the multiconductor transmission line (MTL) per-unit-length (p.u.l.) parameters. In the present paper, the cited method is modified to perform the parametric sensitivity analysis of MTLs with respect to either geometric or physical parameters directly in the time-domain, leading to a macromodel that can be used with both linear and non linear terminations.

This paper is structured as follows. First, an overview of the spectral approach is given in Sections II. The parametric macromodeling strategy in the frequency-domain is shown in Section III, while the time-domain parametric sensitivity analysis is described in Section IV. Finally, two numerical examples are presented in Section V, validating the proposed technique. Conclusions are summed up in Section VI.

\section{Spectral Modeling of Multiconductor TRANSMISSION LINES}

Consider a MTL of length $d$, with $N+1$ conductors and $N \times N$ p.u.l. impedance matrix $\boldsymbol{Z}_{p u l}(s, \boldsymbol{g})$ and $N \times N$ 
p.u.1. admittance matrix $\boldsymbol{Y}_{p u l}(s, \boldsymbol{g})$ [11]. Assuming the quasitransverse electromagnetic (TEM) hypothesis, the propagation of voltages and currents along the line is described by the Telegrapher's equations [11]. Using the dyadic Green's function method proposed in [5], the voltage along the uniform MTL with length $d$ can be evaluated as

$$
\begin{aligned}
\boldsymbol{V}(z, s, \boldsymbol{g}) & =\boldsymbol{G}(z, 0, s, \boldsymbol{g})\left(-\boldsymbol{Z}_{\text {pul }}(s, \boldsymbol{g}) \boldsymbol{I}(0, s, \boldsymbol{g})\right) \\
& +\boldsymbol{G}(z, d, s, \boldsymbol{g})\left(-\boldsymbol{Z}_{\text {pul }}(s, \boldsymbol{g}) \boldsymbol{I}(d, s, \boldsymbol{g})\right)
\end{aligned}
$$

where $s$ is the Laplace variable, $g$ is a vector containing all the geometric or physical parameters of the MTL, while $\boldsymbol{I}(0, s, \boldsymbol{g})$ and $\boldsymbol{I}(d, s, \boldsymbol{g})$ are $N$-vectors containing the MTL port currents at the input and output ports, respectively. The $N \times N$ dyadic Green's function $\boldsymbol{G}\left(z, z^{\prime}, s, \boldsymbol{g}\right)$ for uniform MTLs is written in a spectral form as

$$
\begin{aligned}
\boldsymbol{G}\left(z, z^{\prime}, s, \boldsymbol{g}\right) & =-\sum_{n=0}^{\infty} \boldsymbol{\Psi}_{n}(s, \boldsymbol{g})^{-1} \varphi_{n}(z) \varphi_{n}\left(z^{\prime}\right) \\
\boldsymbol{\Psi}_{n}(s, \boldsymbol{g}) & =\gamma^{2}(s, \boldsymbol{g})+\left(\frac{n \pi}{d}\right)^{2} \boldsymbol{U} \\
\boldsymbol{\gamma}^{2}(s, \boldsymbol{g}) & =\boldsymbol{Z}_{\text {pul }}(s, \boldsymbol{g}) \boldsymbol{Y}_{p u l}(s, \boldsymbol{g}) \\
\varphi_{n}(z) & =A_{n} \cos \left(\frac{n \pi z}{d}\right) \\
A_{n} & =\sqrt{\frac{1}{d}} \text { if } n=0 ; \quad \sqrt{\frac{2}{d}} \text { otherwise }
\end{aligned}
$$

where $\boldsymbol{U}$ is the $N \times N$ identity matrix. Using (1) to calculate the voltage at the MTL ports allows to write

$$
\boldsymbol{V}(s, \boldsymbol{g})=\boldsymbol{Z}(s, \boldsymbol{g}) \boldsymbol{I}(s, \boldsymbol{g})
$$

where $\boldsymbol{V}(s, \boldsymbol{g})$ and $\boldsymbol{I}(s, \boldsymbol{g})$ are the $2 N$-vectors of the voltage and the current at the MTL ports, respectively, and the $2 N \times$ $2 N$ impedance matrix $\boldsymbol{Z}(s, \boldsymbol{g})$ can be expressed with respect to the Green's function [5] as

$$
\boldsymbol{Z}(s, \boldsymbol{g})=\sum_{n=0}^{\infty} \boldsymbol{\Psi}_{n}(s, \boldsymbol{g})^{-1} A_{n}^{2} \boldsymbol{Z}_{p u l}(s, \boldsymbol{g}) \boldsymbol{U}_{n}
$$

The symbol $\boldsymbol{U}_{n}$ represents the $2 N \times 2 N$ matrix

$$
\boldsymbol{U}_{n}=\left[\begin{array}{cc}
\boldsymbol{U} & (-1)^{n} \boldsymbol{U} \\
(-1)^{n} \boldsymbol{U} & \boldsymbol{U}
\end{array}\right]
$$

Referring to [5], each term of the infinite summation (4) is called modal impedance and is represented by the symbol $\boldsymbol{Z}_{n}(s, \boldsymbol{g})$. The series form of the dyadic Green's function is very general; it only assumes that the multiconductor transmission line supports the quasi-TEM mode and is uniform along the $z$-axis. No hypothesis has been done regarding the nature of the p.u.l. impedance $\boldsymbol{Z}_{p u l}(s, \boldsymbol{g})$ and admittance $\boldsymbol{Y}_{p u l}(s, \boldsymbol{g})$ matrices and, as a consequence, on the propagation constant $\gamma^{2}(s, \boldsymbol{g})$. This means that skin-effect, slow-wave effect and dielectric polarization losses can be easily modeled and incorporated in transient analysis once the frequencydependent p.u.l. parameters are available [5].

Equation (3) leads to an analytical expression for the voltage sensitivity with respect to the parameters $\boldsymbol{g}$ at the MTL ports

$$
\widehat{\boldsymbol{V}}(s, \boldsymbol{g})=\widehat{\boldsymbol{Z}}(s, \boldsymbol{g}) \boldsymbol{I}(s, \boldsymbol{g})+\boldsymbol{Z}(s, \boldsymbol{g}) \widehat{\boldsymbol{I}}(s, \boldsymbol{g})
$$

The matrix $\widehat{\boldsymbol{Z}}(s, g)$ can be written as [6]

$$
\begin{aligned}
\widehat{\boldsymbol{Z}}(s, \boldsymbol{g}) & =\sum_{n=0}^{\infty}\left(-\boldsymbol{\Psi}_{n}(s, \boldsymbol{g})^{-1}\right) A_{n}^{2} \widehat{\gamma^{2}}(s, \boldsymbol{g}) \boldsymbol{\Psi}_{n}(s, \boldsymbol{g})^{-1} \\
& +\sum_{n=0}^{\infty} \boldsymbol{\Psi}_{n}(s, g)^{-1} A_{n}^{2} \widehat{\boldsymbol{Z}}_{p u l}(s, \boldsymbol{g}) \boldsymbol{U}_{n}
\end{aligned}
$$

where the propagation constant sensitivity with respect to $g$ is

$$
\widehat{\boldsymbol{\gamma}^{2}}(s, \boldsymbol{g})=\widehat{\boldsymbol{Z}}_{p u l}(s, \boldsymbol{g}) \boldsymbol{Y}_{p u l}(s, \boldsymbol{g})+\boldsymbol{Z}_{p u l}(s, \boldsymbol{g}) \widehat{\boldsymbol{Y}}_{p u l}(s, \boldsymbol{g})
$$

Each term of the infinite summation (6) can be represented with the symbol $\widehat{\boldsymbol{Z}}_{n}(s, \boldsymbol{g})$ since it is the sensitivity with respect to $\boldsymbol{g}$ of the corresponding modal impedance $\boldsymbol{Z}_{n}(s, \boldsymbol{g})$ [6].

Note that only the matrices $\boldsymbol{Z}_{p u l}(s, \boldsymbol{g}), \boldsymbol{Y}_{p u l}(s, \boldsymbol{g})$ and the corresponding derivatives with respect to parameters $\boldsymbol{g}$ (i.e., the $\widehat{\boldsymbol{Z}}_{p u l}(s, \boldsymbol{g})$ and $\widehat{\boldsymbol{Y}}_{p u l}(s, \boldsymbol{g})$ matrices) are required to obtain $\widehat{\boldsymbol{Z}}(s, \boldsymbol{g})$ from (6). While the p.u.l. parameters can be evaluated through direct measurements [12] on the MTL or using full-wave electromagnetic simulators, information on their derivatives are not a priori known. A key aspect of the proposed technique is to find a good approximation for the $\widehat{\boldsymbol{Z}}_{\text {pul }}(s, \boldsymbol{g})$ and $\widehat{\boldsymbol{Y}}_{\text {pul }}(s, \boldsymbol{g})$ matrices in the entire design space.

Note that relations (4) and (6) give a closed form expression for the $\boldsymbol{Z}(s, \boldsymbol{g})$ and $\widehat{\boldsymbol{Z}}(s, \boldsymbol{g})$ matrix, respectively, with respect to the p.u.l. parameters $\boldsymbol{Z}_{p u l}(s, \boldsymbol{g})$ and $\boldsymbol{Y}_{p u l}(s, \boldsymbol{g})$ and the corresponding sensitivity $\widehat{\boldsymbol{Z}}_{\text {pul }}(s, \boldsymbol{g})$ and $\widehat{\boldsymbol{Y}}_{\text {pul }}(s, \boldsymbol{g})$, but both expressions require to perform an infinite summation. However, only a finite number of modes is needed to model the impedance matrix and its sensitivity accurately over the frequency bandwidth of interest, as shown in [3]-[6]. Hence a mode-selection criterion must be used to calculate (4) and (6) for all the values of $\boldsymbol{g}$ in the design space.

\section{PARAMETRIC MACROMODELING OF MULTICONDUCTOR TRANSMISSION LINES}

In this section, it is shown that the spectral decomposition [5], [6] allows to calculate a rational model of $\boldsymbol{Z}(s, \boldsymbol{g})$ and $\widehat{\boldsymbol{Z}}(s, \boldsymbol{g})$ for all the values of $\boldsymbol{g}$ in the design space.

To attain this goal, we start from the p.u.l. impedance and admittance matrices evaluated for a discrete set of values of the parameters $\boldsymbol{g}$ in the design space. The p.u.l. impedance and admittance are usually smooth functions with respect to frequency and physical or geometrical parameters and the corresponding matrices are symmetric, thus the initial data for the p.u.l. impedance and admittance matrices can be easily numerically interpolated leading to accurate estimation of $\boldsymbol{Z}_{p u l}(s, \boldsymbol{g})$ and $\boldsymbol{Y}_{p u l}(s, \boldsymbol{g})$ in the entire design space with very small computational cost. The use of continuously differentiable interpolation schemes allows to obtain also the $\widehat{\boldsymbol{Z}}_{p u l}(s, \boldsymbol{g})$ and $\widehat{\boldsymbol{Y}}_{\text {pul }}(s, \boldsymbol{g})$ matrices in the entire design space with respect to physical or geometrical parameters $\boldsymbol{g}$ of the interpolating function. Therefore, we have computed parametric macromodels of the p.u.l. parameters and corresponding sensitivities with respect to $(s, \boldsymbol{g})$.

Next, we switch from multivariate models to univariate models fixing the value of the parameters: $\boldsymbol{g}=\overline{\boldsymbol{g}}$. Then, the 
matrices $\boldsymbol{Z}(s, \overline{\boldsymbol{g}})$ and $\widehat{\boldsymbol{Z}}(s, \overline{\boldsymbol{g}})$ can be calculated using the spectral decomposition (4) and (6), once the infinite summation of modes is truncated. Algorithm 1 describes the proposed adaptive criterion to choose the required number of modes $M$ for the different values of the geometrical or physical parameters, indicating with $\overline{\boldsymbol{g}}$ a fixed set of parameters $\boldsymbol{g}$ and with $\widehat{\boldsymbol{Z}}_{n}(s, \overline{\boldsymbol{g}})$ the corresponding sensitivity modal impedance. The adopted solution is shown only for the $\widehat{Z}(s, \bar{g})$ matrix, since a similar procedure for the impedance matrix $\boldsymbol{Z}(s, \overline{\boldsymbol{g}})$ is applied.

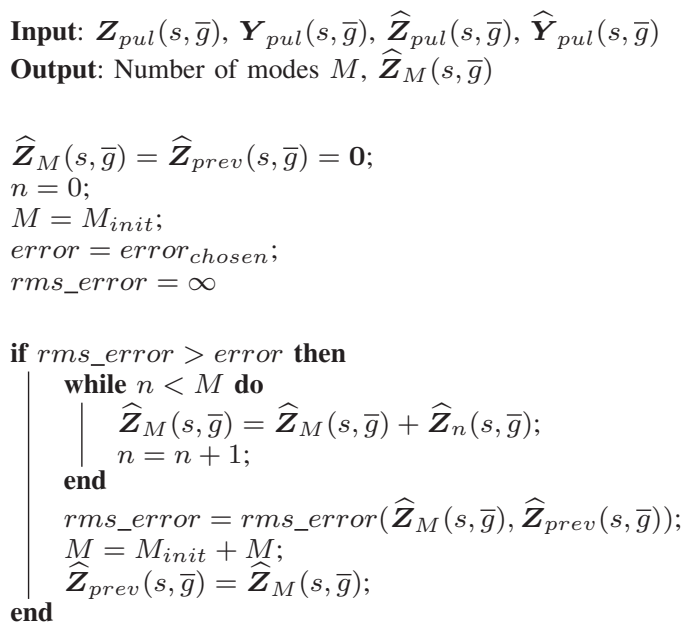

Algorithm 1: Adaptive mode selection strategy.

The desired stable rational models for $\boldsymbol{Z}(s, \overline{\boldsymbol{g}})$ and $\widehat{\boldsymbol{Z}}(s, \overline{\boldsymbol{g}})$ are now obtained using the Vector Fitting algorithm [13], [14]. Finally, the passivity of the model for the impedance matrix can be checked and enforced in a post-processing step by means of standard techniques [15], [16]. To perform design activities that need multiple simulations (e.g. design space exploration, optimization, sensitivity analysis), for each value $\overline{\boldsymbol{g}}$ of interest the parametric macromodels of the p.u.l. parameters are evaluated and the related rational macromodels of $\boldsymbol{Z}(s, \overline{\boldsymbol{g}})$ and $\widehat{\boldsymbol{Z}}(s, \overline{\boldsymbol{g}})$ are computed. The proposed parametric macromodeling strategy is summarized in Fig. 1.

Note that the desired rational model for the impedance matrix could be calculated as explained in [5]. The combination of a rational model for the p.u.l. parameters with the Green's function, used to expand the solution of the SturmLiouville problem, allows to compute poles and residues of the impedance matrix independently for each mode, reducing the complexity of the system identification significantly. In fact, the technique described in [3], [4] first builds parametric macromodels for the p.u.l. parameters or for the modal impedance, using the Multivariate Orthonormal Vector Fitting Technique (MOVF) [17], then it combines the initial macromodels according to (4) to obtain the desired macromodel for the impedance matrix $\boldsymbol{Z}(s, \boldsymbol{g})$ in the entire design space. Our goal, however, it is to build a parametric macromodel also for the $\widehat{Z}(s, \boldsymbol{g})$ matrix, to be able to perform a parametric sensitivity analysis. Using the same approach as described above for the spectral decomposition of $\widehat{\boldsymbol{Z}}(s, \boldsymbol{g})$ may be inefficient. It is easy to see that computing each mode of (6) is more expensive than computing a mode of (4). If, for each value of interest of $\boldsymbol{g}$, a large number of modes is needed to compute $\widehat{\boldsymbol{Z}}(s, \boldsymbol{g})$ in the frequency band of interest, the corresponding rational model would have a high order of poles if (6) is calculated starting from a rational model for the p.u.1. parameters and the corresponding sensitivities. In the proposed approach, the number of poles needed for the rational model of the matrix $\widehat{\boldsymbol{Z}}(s, \boldsymbol{g})$ depends only on its frequency behavior, leading to an efficient calculation of the spectral decomposition (6).

Note that the $\widehat{\boldsymbol{Z}}(s, \boldsymbol{g})$ can also be evaluated with perturbation-based techniques, since the computation of the $\boldsymbol{Z}(s, \boldsymbol{g})$ matrix can be efficiently performed using the exact transmission line theory. In both approaches an approximation is introduced in the sensitivity computation, but the p.u.l. parameters $\boldsymbol{Z}_{p u l}(s, \boldsymbol{g})$ and $\boldsymbol{Y}_{p u l}(s, \boldsymbol{g})$ are smoother functions with respect to physical or geometrical parameters than the MTL impedance $\boldsymbol{Z}(s, \boldsymbol{g})$, affecting the accuracy of the numerical approximation. Furthermore, the proposed macromodeling technique uses the spectral decomposition (6) to express the sensitivity of the MTL impedance matrix in a closed form, using a numerical approximation only for the calculation of matrices $\widehat{\boldsymbol{Z}}(s, \boldsymbol{g})$ and $\widehat{\boldsymbol{Y}}(s, \boldsymbol{g})$, while sensitivity perturbationbased techniques are prone to inaccuracies depending on the magnitude of the perturbation [18].

\section{TIME-DOMAIN PARAMETRIC SENSITIVITY ANALYSIS}

The proposed technique represents both $\boldsymbol{Z}(s, \boldsymbol{g})$ and $\widehat{Z}(s, g)$ with a rational model for each desired value of $\boldsymbol{g}$ in the design space. The generation of the time-domain statespace equations in the Jordan form [18], [19] is straightforward. In fact, based on (3), it is easy to calculate the timedomain model for the voltage at the MTL ports

$$
\begin{aligned}
& \dot{\boldsymbol{x}}(t)=\boldsymbol{A}_{Z} \boldsymbol{x}(t)+\boldsymbol{B}_{Z} \boldsymbol{i}(t) \\
& \boldsymbol{v}(t)=\boldsymbol{C}_{Z} \boldsymbol{x}(t)+\boldsymbol{D}_{Z} \boldsymbol{i}(t)
\end{aligned}
$$

Hence, based on equation (5), the voltage sensitivity can be represented by two different sets of state-space equations

$$
\begin{aligned}
\dot{\boldsymbol{x}}_{1}(t) & =\boldsymbol{A}_{\widehat{Z}} \boldsymbol{x}_{1}(t)+\boldsymbol{B}_{\widehat{Z}} \boldsymbol{i}(t) \\
\widehat{\boldsymbol{v}}_{1}(t) & =\boldsymbol{C}_{\widehat{Z}} \boldsymbol{x}_{1}(t)+\boldsymbol{D}_{\widehat{Z}} \boldsymbol{i}(t) \\
\dot{\boldsymbol{x}}_{2}(t) & =\boldsymbol{A}_{Z} \boldsymbol{x}_{2}(t)+\boldsymbol{B}_{Z} \widehat{\boldsymbol{i}}(t) \\
\widehat{\boldsymbol{v}}_{2}(t) & =\boldsymbol{C}_{Z} \boldsymbol{x}_{2}(t)+\boldsymbol{D}_{Z} \widehat{\boldsymbol{i}}(t)
\end{aligned}
$$

where $\boldsymbol{x}(t), \boldsymbol{x}_{1}(t)$ and $\boldsymbol{x}_{2}(t)$ are state-space variables, while $\boldsymbol{i}(t)=\left[\begin{array}{ll}\boldsymbol{i}_{0}(t) & \boldsymbol{i}_{d}(t)\end{array}\right]^{T}$ and $\widehat{\boldsymbol{i}}(t)=\left[\begin{array}{ll}\boldsymbol{i}_{0}(t) & \boldsymbol{i}_{d}(t)\end{array}\right]^{T}$ are the inputs [18]. Line terminations are assumed to be modeled by current sources $i_{S}(t)$ and voltage-driven lumped linear and nonlinear elements that can be described by the following equation

$$
\boldsymbol{i}(t)=\boldsymbol{i}_{S}(t)-\boldsymbol{G} \boldsymbol{v}(t)-\boldsymbol{C} \frac{d \boldsymbol{v}(t)}{d t}-\boldsymbol{f}(\boldsymbol{v}(t))
$$

where $\boldsymbol{v}(t)$ and $\boldsymbol{i}(t)$ are the port voltages and currents, matrices $\boldsymbol{G}$ and $\boldsymbol{C}$ describe linear resistive and capacitive lumped elements respectively, and $\boldsymbol{f}(\boldsymbol{v}(t))$ describes lumped nonlinear components. Calculating the derivative of (11) with respect to 


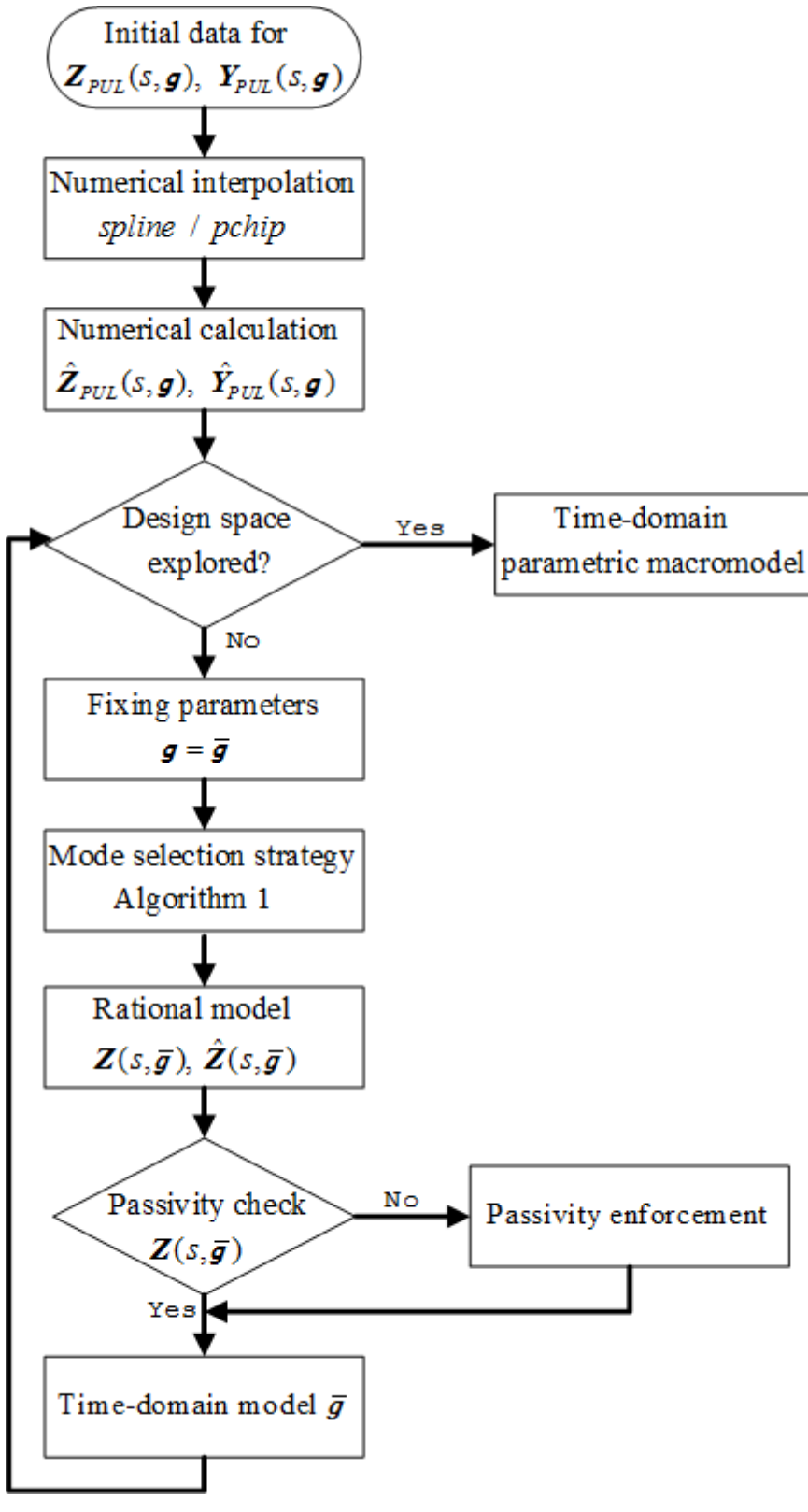

Fig. 1. Description of the parametric macromodeling strategy.

the parameters $g$, it is possible to obtain the expression of sensitivity of the terminations

$$
\widehat{\boldsymbol{i}}(t)=-\boldsymbol{G} \widehat{\boldsymbol{v}}(t)-\boldsymbol{C} \frac{d \widehat{\boldsymbol{v}}(t)}{d t}-\frac{d \boldsymbol{f}(\boldsymbol{v}(t))}{d \boldsymbol{v}(t)} \widehat{\boldsymbol{v}}(t)
$$

More complex termination networks can be incorporated by using the modified nodal analysis (MNA) [20]. The port voltage sensitivity can be finally expressed as

$$
\widehat{\boldsymbol{v}}(t)=\widehat{\boldsymbol{v}}_{1}(t)+\widehat{\boldsymbol{v}}_{2}(t)
$$

Once the port currents are evaluated [18] by solving (8) and (11), state variables $\boldsymbol{x}_{1}, \boldsymbol{x}_{2}$ and current sensitivity are obtained through (9), (10), (11), (12) and (13). The port voltage sensitivities are finally recovered using (13).

The proposed macromodeling technique describes an uniform MTL with state-space representation in the time-domain as function of a set of physical or geometrical parameters $\boldsymbol{g}$. The macromodel gives accurate sensitivity information at the ports of the lines with respect to $\boldsymbol{g}$ and allows to include non-linear terminations during the time-domain analysis. A multistep procedure is used to calculate the state-space model for each value of the physical or geometrical parameters in the design space, see Fig. 1, making it suitable to be used in an optimization process. Equations (8)-(13) must be solved separately for each time-domain model calculated for particular combination $\bar{g}$ of the geometrical or physical parameters, making the proposed technique efficient if the number of parameters taken into account is limited.

\section{NumERICAL EXAMPLES}

In this section the presented technique is applied to different uniform MTLs. In each example the $\widehat{\boldsymbol{Z}}_{p u l}$ and $\widehat{\boldsymbol{Y}}_{p u l}$ matrices are interpolated by two polynomial techniques: spline and pchip [21], [22]. pchip finds values of an underlying interpolant function $P(x) \in C^{1}$ at intermediate points under the constraint that in each subinterval $P(x)$ is the cubic Hermite interpolant, thereby preserving shape and monotonicity of the data. spline returns the polynomial form of the cubic spline interpolant $S(x) \in C^{2}$ calculated at the data points, resulting in a not necessarily monotonic interpolation. To describe the accuracy of the parametric macromodeling strategy in the frequency-domain, for each example the maximum value of the Frobenius norm of the relative error, indicated with $R M S_{\text {weighted }}$, between the two $\widehat{\boldsymbol{Z}}(s, \boldsymbol{g})$ macromodels over the validation grid and the $R M S_{\text {weighted }}$ error between the macromodel of the $\boldsymbol{Z}(s, \boldsymbol{g})$ matrix and its computation from the exact transmission line theory (TLT) will be shown. To validate the proposed method, port voltages and currents are calculated using the classic transmission line theory (TLTIFFT) in the case of linear terminations and by means of a solver for ordinary differential equations (TLT-NLS) in the case of non-linear terminations. The corresponding sensitivities are obtained using the perturbative approach. These signals are compared with the ones of the MTL time-domain macromodel obtained with the newly proposed method, where both the polynomial techniques spline and pchip are used to build the macromodel.

\section{A. Three-conductor transmission line with non-linear termi- nations}

In the first example, two coplanar microstrips over a ground plane (length $d=10 \mathrm{~cm}$ ) with frequency-dependent p.u.l. parameters have been modeled within the frequency range [100 $\mathrm{kHz}-10 \mathrm{GHz}$ ], see Fig. 2.

The conductors have width $w=100 \mu m$ and thickness $t=50 \mu \mathrm{m}$. The spacing $S$ between the microstrips varies over the design range $[100-500] \mu m$. The dielectric is $300 \mu m$ thick and it is characterized by a dispersive and lossy permittivity which has been modeled by the wideband Debye model [23]. The frequency-dependent p.u.l. parameters are evaluated using a commercial tool [24] over a reference grid of $250 \times 20$ samples, for frequency and spacing respectively, and the validation is performed over a grid of 10 spacing 


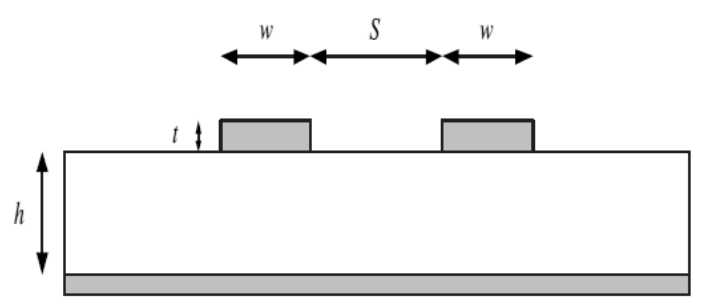

Fig. 2. Cross section of the two coupled microstrip.

samples that have not been used for the generation of the macromodel. The accuracy of the parametric macromodeling strategy is good, as can be assessed in the frequency-domain from Table I.

TABLE I

MAXIMUM PARAMETRIC MACROMODELING ERROR

\begin{tabular}{|l|c|}
\hline Macromodel & $R M S_{\text {weighted }}$ \\
\hline $\boldsymbol{Z}(s, \boldsymbol{g})$ spline & 0.0021 \\
$\boldsymbol{Z}(s, \boldsymbol{g})$ pchip & 0.0021 \\
$\widehat{\boldsymbol{Z}}(s, \boldsymbol{g})$ & 0.0644 \\
\hline
\end{tabular}

The time-domain simulations are performed with the following settings: one line is excited by a voltage pulse with amplitude $1 \mathrm{~V}$, rise/fall times $900 \mathrm{ps}$, width $2 \mathrm{~ns}$, initial delay $7 n s$ and internal resistance $R_{S}=50 \Omega$. The driven line is terminated on a direct biased diode. The current of the diode is given by

$$
i_{D}(t)=I_{0}\left(e^{\frac{v(t)}{V_{T}}}-1\right)
$$

where $I_{0}=10 \mathrm{nA}$ and $V_{T}=25 \mathrm{mV}$. The victim line is terminated on the near-end by $R_{N E}=50 \Omega$ and on the far-end by a direct biased diode, described by (14). Figs. 3 and 4 show the accuracy of the proposed technique in the time-domain using the pchip interpolation. Similar results are obtained for the macromodel built using spline.

\section{B. Coupled inverted embedded microstrip lines with} frequency-dependent p.u.l. parameters and linear terminations

In this next example a three conductors transmission line (length $d=1 \mathrm{~mm}$ ) with frequency-dependent p.u.l. parameters has been modeled within the frequency range $[0-$ 200] $G H z$, see Fig. 5. The two inverted microstrips are embedded in a layered background medium, consisting of a doped Silicon substrate with thickness $30 \mu \mathrm{m}$, relative permittivity $\epsilon_{r}=11.7$, conductivity $\sigma=10 \mathrm{~S} / \mathrm{m}$, and an insulator, being $11.4 \mu \mathrm{m}$ thick $\mathrm{SiO}_{2}$ with relative permittivity $\epsilon_{r}=3.9$ and loss tangent $\tan \delta=0.001$ [25]. On the top of insulator the Aluminum ground plate of thickness $3 \mu \mathrm{m}$ is found. The Aluminum has a conductivity of $3.77 \cdot 10^{7} \mathrm{~S} / \mathrm{m}$. The conductors, placed at a distance of $6.4 \mu \mathrm{m}$ above the semiconductor, are also made of Aluminum and have width $2 \mu \mathrm{m}$; the spacing $S \in[1-6] \mu m$ and the height $H \in[1-3] \mu m$ of the conductors are considered design parameters in addition

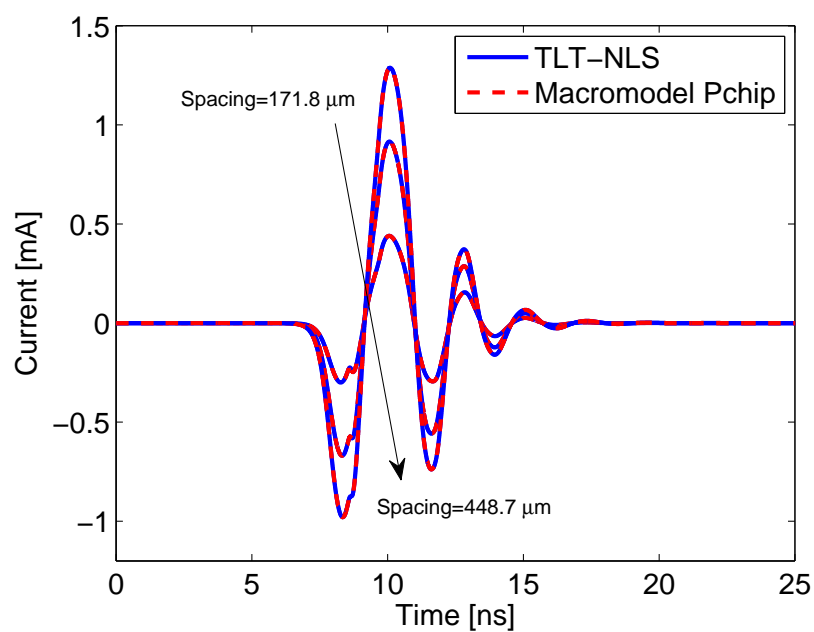

Fig. 3. Example A. Current at the input port of the victim line for $S=[171.8,253.8,448.7] \mu m$.

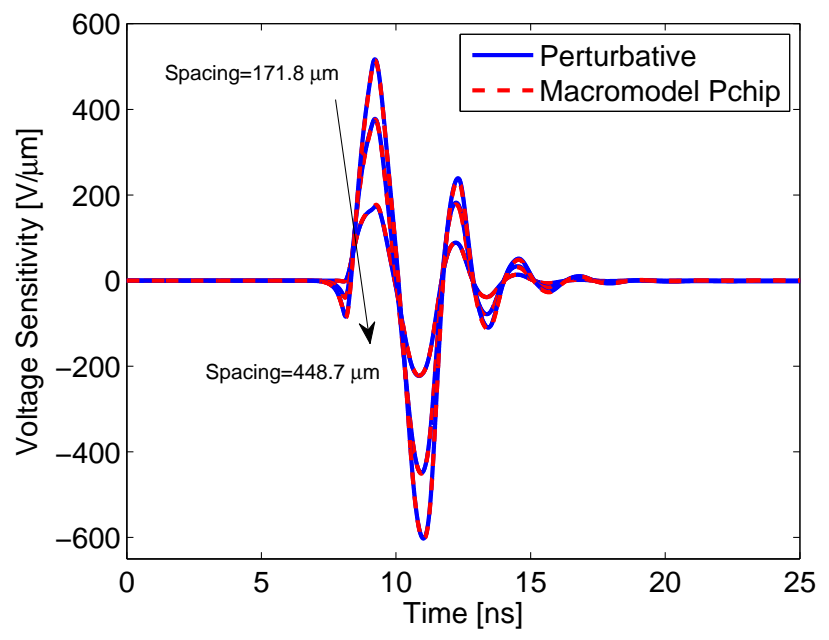

Fig. 4. Example A. Voltage sensitivity at the output port of the victim line for $S=[171.8,253.8,448.7] \mu m$.

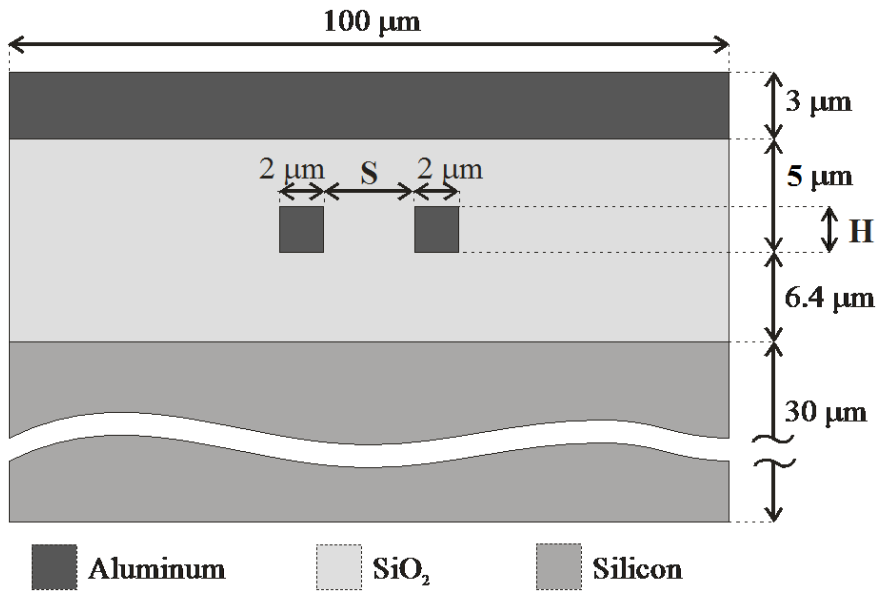

Fig. 5. Cross section of the two inverted coupled microstrip. 
to frequency. The frequency-dependent p.u.l. parameters are evaluated using the technique presented in [26], [27] over a reference grid of $101 \times 11 \times 11$ samples, for frequency, spacing and height respectively. The validation is performed over a grid of $10 \times 10$ samples for spacing and height, that have not been used for the generation of the macromodel. Table II shows the accuracy of the proposed technique in the frequency-domain.

TABLE II

MAXIMUM PARAMETRIC MACROMOdELING ERROR

\begin{tabular}{|l|c|c|}
\hline Macromodel & $R M S_{\text {weighted }}$ Spline & $R M S_{\text {weighted }}$ Pchip \\
\hline $\boldsymbol{Z}(s, \boldsymbol{g})$ & 0.0053 & 0.0033 \\
\hline Macromodel & $R M S_{\text {weighted }}$ Spacing & $R M S_{\text {weighted }}$ Height \\
\hline$\widehat{\boldsymbol{Z}}(s, \boldsymbol{g})$ & 0.0484 & 0.0059 \\
\hline
\end{tabular}

One line is excited by a voltage pulse with amplitude $1 \mathrm{~V}$, rise/fall times $30 \mathrm{ps}$, width $90 \mathrm{ps}$, initial delay $100 \mathrm{ps}$ and internal resistance $R_{S}=1 \Omega$. The driven line is terminated on a parallel RC load $R_{L}=10 \mathrm{k} \Omega, C_{L}=1 \mathrm{pF}$. The victim line is terminated on the near-end by $R_{N E}=1 \Omega$ and on the farend by a parallel RC load $R_{F E}=10 \mathrm{k} \Omega$ and $C_{F E}=1 \mathrm{pF}$. Figs. $6-8$ demonstrate the high accuracy in the time-domain of the proposed method using the spline interpolation for this on-chip example that exhibits a highly dynamic behavior. Similar results are obtained for the macromodel built using pchip.

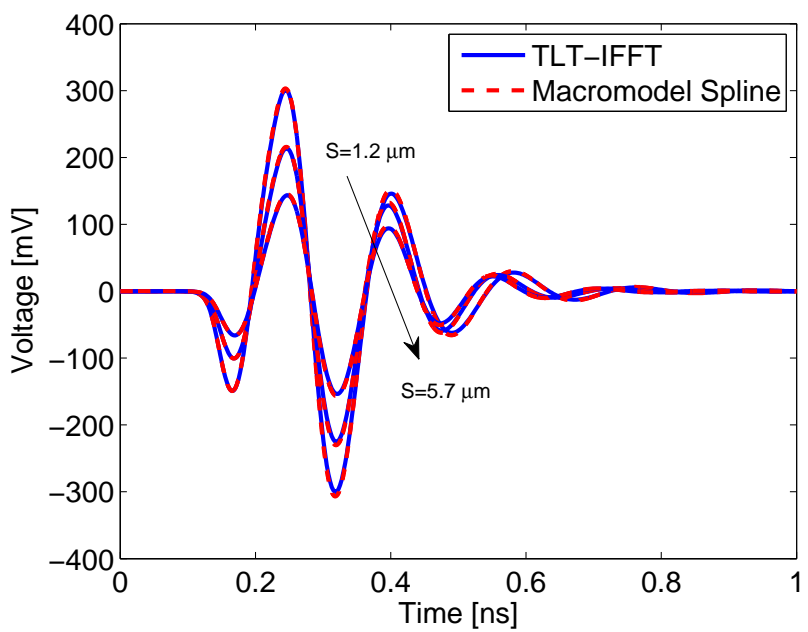

Fig. 6. Example B. Voltage at the output port of the victim line for $S=[1.2,3.3,5.7] \mu m$ and $H=2.3 \mu \mathrm{m}$

\section{CONCLusions}

In this paper, an innovative parametric macromodeling approach for lossy and dispersive MTLs is presented. The dyadic Green's function of the 1-D wave propagation problem is used to compute a spectral decomposition for the voltage sensitivity and this leads to a time-domain macromodel in state-space form. Using standard realization techniques, this macromodel can be easily embedded into conventional

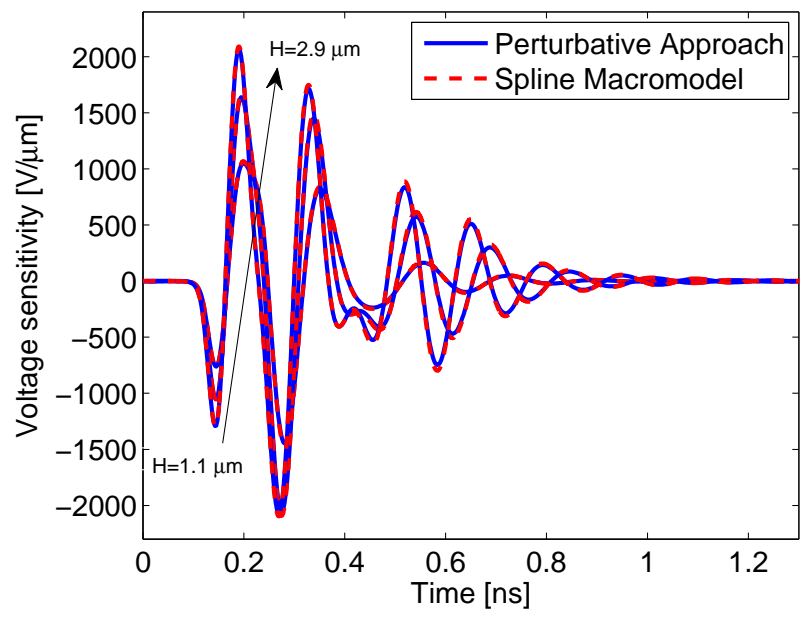

Fig. 7. Example B. Voltage sensitivity with respect to the spacing, at the input port of the victim line for $H=[1.1,1.9,2.9] \mu \mathrm{m}$ and $S=2.8 \mu \mathrm{m}$.

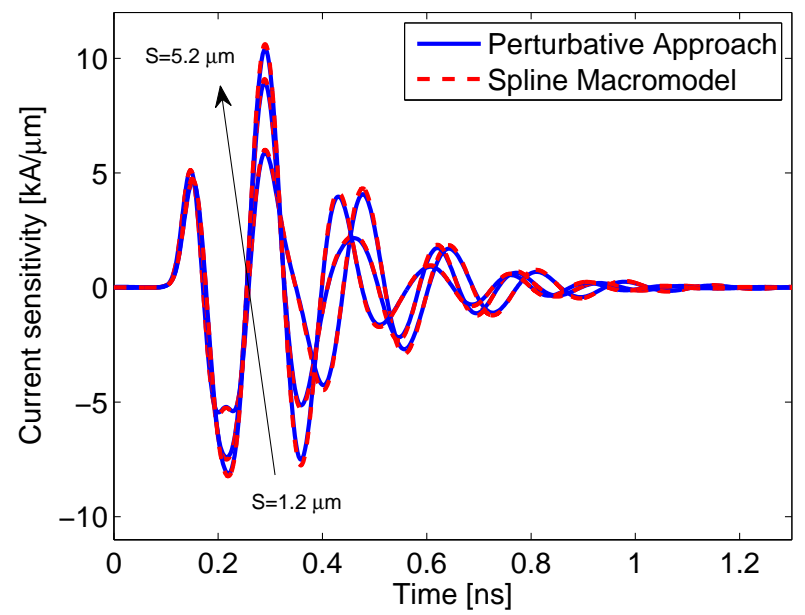

Fig. 8. Example B. Current sensitivity with respect to the height, at the input port of the driven line for $S=[1.2,3.3,5.2] \mu \mathrm{m}$ and $H=2.1 \mu \mathrm{m}$.

SPICE-like solvers. Numerical interpolation of the p.u.l. parameters and evaluation, performed with numerical techniques, of the corresponding sensitivity with respect to physical or geometrical parameters, are used to calculate the spectral decomposition over the entire design space. This leads to a parametric sensitivity analysis that avoids any similarity transformation and incorporates non-linear terminations in a straightforward way. The new proposed technique is validated by comparing the MTL port currents and voltages with classic approaches (TLT-IFFT) and (TLT-NLS). Time-domain simulations confirm reliability and robustness of the proposed method.

\section{REFERENCES}

[1] A. Deutsch, "Electrical characteristics of interconnects for highperformance systems," Proc. IEEE, invited paper, vol. 86, no. 2, pp. 315-355, 1998.

[2] M. Nakhla and R. Achar, Handbook on VLSI. Boca Raton, FL: CRC, 2000. 
[3] F. Ferranti, G. Antonini, T. Dhaene and L. Knockaert, "Parametric macromodeling of lossy and dispersive multiconductor transmission lines," IEEE Trans. Adv. Packag., vol. 33, no. 2, pp. 481-491, May 2010.

[4] F. Ferranti, T. Dhaene, L. Knockaert, and G. Antonini, "Parameterized models for crosstalk analysis in high-speed interconnects," in IEEE International Symposium on Electromagnetic Compatibility 2009, EMC 2009., Aug. 2009, pp. $180-185$.

[5] G. Antonini, "A dyadic Green's function based method for the transient analysis of lossy and dispersive multiconductor transmission lines," IEEE Trans. Microw. Theory Tech., vol. 56, no. 4, pp. 880-895, Apr. 2008.

[6] G. Antonini, L. De Camillis, and F. Ruscitti, "A spectral approach to frequency-domain sensitivity analysis of multiconductor transmission lines," IEEE Microw. Wireless Compon. Lett., pp. 65-67, Feb. 2009.

[7] Jun-Fa Mao, E.S. Kuh, "Fast simulation and sensitivity analysis of lossy transmission lines by the method of characteristics," Circuits and Systems I: Fundamental Theory and Applications, IEEE Transactions on, vol. 44, no. 5, pp. 391-401, May 1997.

[8] N. M. Nakhla, A. Dounavis, M. S. Nakhla, and R. Achar, "Delayextraction-based sensitivity analysis of multiconductor transmission lines with nonlinear terminations," IEEE Trans. Microw. Theory Tech., vol. 53, no. 11, pp. 3520-3530, Nov. 2005.

[9] R. Achar, M. Nakhla, "Simulation of high-speed interconnects," Proc. IEEE, vol. 89, no. 5, pp. 693-728, May 2001.

[10] G. Antonini, "Spice equivalent circuits of frequency-domain responses," IEEE Trans. Electromagn. Compat., vol. 45, no. 3, pp. 502-512, 2003.

[11] C. R. Paul, Analysis of Multiconductor Transmission Lines, 2nd ed. New York, NY: John Wiley \& Sons, 2008.

[12] L. Knockaert, D. De Zutter, F. Olyslager, E. Laermans, J. De Geest, "Recovering lossy multiconductor transmission line parameters from impedance or scattering representations," IEEE Trans. Adv. Packag., vol. 25, no. 2, pp. 200-205, May 2002.

[13] B. Gustavsen and A. Semlyen, "Rational approximation of frequency domain responses by vector fitting," IEEE Trans. Power Del., vol. 14, no. 3, pp. 1052-1061, Jul. 1999.

[14] D. Deschrijver, M.Mrozowski, T. Dhaene, and D. De Zutter, "Macromodeling of multiport systems using a fast implementation of the vector fitting method," IEEE Microw. Wireless Compon. Lett., vol. 18, no. 6, pp. 383-385, June 2008.

[15] B. Gustavsen, "Fast passivity enforcement for pole-residue models by perturbation of residue matrix eigenvalues," IEEE Trans. Power Del., vol. 23, no. 4, pp. 2278-2285, Oct. 2008.

[16] A. Semlyen and B. Gustavsen, "A half-size singularity test matrix for fast and reliable passivity assessment of rational models," IEEE Trans. Power Del., vol. 24, no. 1, pp. pp. 345-351, Jan. 2009.

[17] D. Deschrijver, T. Dhaene and D. De Zutter, "Robust parametric macromodeling using multivariate orthonormal vector fitting," IEEE Trans. Microw. Theory Tech., vol. 56, no. 7, pp. 1661-1667, Jul. 2008.

[18] G. Antonini and L. de Camillis, "Time-domain Green's function-based sensitivity analysis of multiconductor transmission lines with nonlinear terminations," IEEE Microw. Wireless Compon. Lett., vol. 19, no. 7, Jul. 2009.

[19] C.-T. Chen, Linear System Theory and Design, ser. Electrical and Computer Engineering. New York, NY: Oxford University Press, 1998.

[20] C. Ho, A. Ruehli, and P. Brennan, "The modified nodal approach to network analysis," IEEE Transactions on Circuits and Systems, vol. 22, no. 6, pp. 504-509, June 1975.

[21] C. de Boor, A Practical Guide to Splines. Springer-Verlag, 1978.

[22] F. N. Fritsch and R. E. Carlson, "Monotone piecewise cubic interpolation," SIAM J. Numerical Analysis, vol. 17, pp. 238-246, Nov. 1980.

[23] A. R. Djordjevic̀, R.M. Biljic̀, V.D. Likar-Smiljanic̀, T.K. Sarkar, "Wideband frequency-domain characterization of fr-4 and time-domain causality," IEEE Trans. Electromagn. Compat., vol. 43, no. 4, pp. 662667, Nov. 2001

[24] Simbeor, Electromagnetic Simulation Environment with 3D Full-Wave Field Solver for Multi-Layered Circuits. Seattle: Simberian Inc.

[25] D. Vande Ginste and D. De Zutter, "Influence of the trapezoidal crosssection of single and coupled inverted embedded microstrip lines on signal integrity," in General Assembly and Scientific Symposium, 2011 XXXth URSI, Aug. 2011, pp. $1-4$.

[26] T. Demeester and D. De Zutter, "Quasi-TM transmission line parameters of coupled lossy lines based on the Dirichlet to Neumann boundary operator," IEEE Trans. Microw. Theory Tech., vol. 56, no. 7, pp. 16491660, Jul. 2008.
[27] T. Demeester, D.Vande Ginste and D. De Zutter, "Accurate study of the electromagnetic and circuit behavior of finite conducting wedges and interconnects with arbitrary cross-sections," IEEE 19th EPEPS, Austin, Texas, USA, pp. 133-136, Oct. 2010.

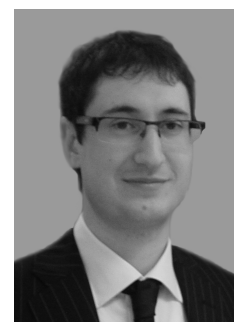

Domenico Spina received the M.S. degree (summa cum laude) in electronic engineering from the Università degli Studi dell'Aquila in 2010. Since October 2010, he has been pursuing the Ph.D. in the Department of Information Technology (INTEC) at Ghent University in Belgium. His research interests include modeling and simulation, system identification, microwave engineering and sensitivity analysis.

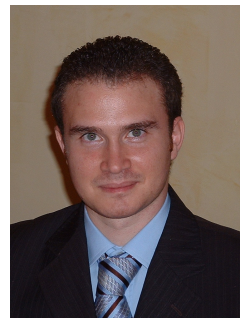

Francesco Ferranti (M'10) received the B.S. degree (summa cum laude) in electronic engineering from the Università degli Studi di Palermo, Palermo, Italy, in 2005, the M.S. degree (summa cum laude and honors) in electronic engineering from the Università degli Studi dell'Aquila, L'Aquila, Italy, in 2007, and the Ph.D. degree in electrical engineering from the University of Ghent, Ghent, Belgium, in 2011. $\mathrm{He}$ is currently a Post-Doctoral Research Fellow with the Department of Information Technology (INTEC), Ghent University, Ghent, Belgium. His research interests include parametric macromodeling, parameterized model order reduction, electromagnetic compatibility numerical modeling, system identification.

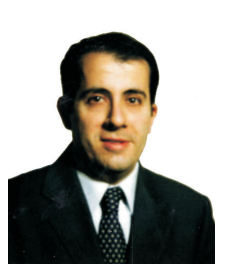

Giulio Antonini (M'94-SM'05) received the Laurea degree (summa cum laude) in electrical engineering from the Università degli Studi dell'Aquila, in 1994, and the Ph.D. degree in electrical engineering from the University of Rome "La Sapienza," in 1998. Since 1998, he has been with the UAq EMC Laboratory, Department of Electrical Engineering, University of L'Aquila, where he is currently Associate Professor. His research interests focus on EMC analysis, numerical modeling, and in the field of signal integrity for high-speed digital systems. He has authored or coauthored more than 180 technical papers and two book chapters. Furthermore, he has given keynote lectures and chaired several special sessions at international conferences. He holds one European patent. Dr. Antonini was the recipient of the IEEE TRANSACTIONS ON ELECTROMAGNETIC COMPATIBILITY Best Paper Award in 1997, the CST University Publication Award in 2004, the IBM Shared University Research Award in 2004, 2005, and 2006. In 2006, he received a Technical Achievement Award from the IEEE EMC Society "for innovative contributions to computational electromagnetic on the Partial Element Equivalent Circuit (PEEC) technique for EMC applications." He also received the IET-SMT Best Paper Award in 2008. He is vice-chairman of the dell'IEEE EMC Italy Chapter, member of the TC-9 committee, and vice-chairman of the TC-10 Committee of the IEEE EMC Society. He serves as member of the editorial board of IET Science, Measurements, and Technology. He serves as reviewer in a number of IEEE journals. 


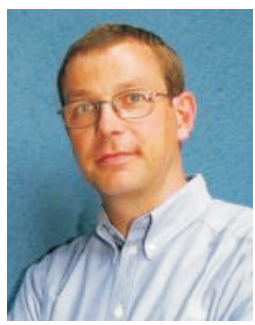

Tom Dhaene was born in Deinze, Belgium, on June 25, 1966. He received the Ph.D. degree in electrotechnical engineering from the University of Ghent, Ghent, Belgium, in 1993. From 1989 to 1993, he was Research Assistant at the University of Ghent, in the Department of Information Technology, where his research focused on different aspects of full-wave electro-magnetic circuit modeling, transient simulation, and time-domain characterization of high-frequency and high-speed interconnections. In 1993, he joined the EDA company Alphabit (now part of Agilent). He was one of the key developers of the planar EM simulator ADS Momentum. Since September 2000, he has been a Professor in the Department of Mathematics and Computer Science at the University of Antwerp, Antwerp, Belgium. Since October 2007, he is a Full Professor in the Department of Information Technology (INTEC) at Ghent University, Ghent, Belgium. As author or co-author, he has contributed to more than 150 peer-reviewed papers and abstracts in international conference proceedings, journals and books. He is the holder of 3 US patents.

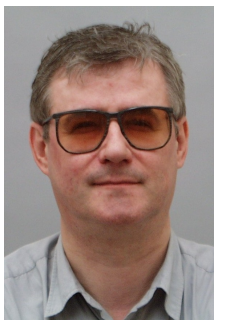

Luc Knockaert received the M. Sc. Degree in physical engineering, the M. Sc. Degree in telecommunications engineering and the $\mathrm{Ph}$. D. Degree in electrical engineering from Ghent University, Belgium, in 1974, 1977 and 1987, respectively. From 1979 to 1984 and from 1988 to 1995 he was working in North-South cooperation and development projects at the Universities of the Democratic Republic of the Congo and Burundi. He is presently affiliated with the Interdisciplinary Institute for BroadBand Technologies (www.ibbt.be) and a professor at the Dept. of Information Technology, Ghent University (www.intec.ugent.be). His current interests are the application of linear algebra and adaptive methods in signal estimation, model order reduction and computational electromagnetics. As author or co-author he has contributed to more than 100 international journal and conference publications. He is a member of MAA, SIAM and a senior member of IEEE.

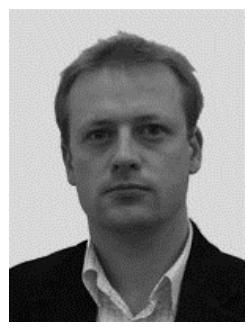

Dries Vande Ginste was born in 1977. He received the M.S. degree and the Ph.D. degree in electrical engineering from Ghent University, Gent, Belgium, in 2000 and 2005, respectively. He is currently an Assistent Professor with the Electromagnetics Group, Department of Information Technology, Ghent University. In June and July 2004, he was a Visiting Scientist at the Department of Electrical and Computer Engineering, University of Illinois at UrbanaChampaign (UIUC), IL, USA. From September to November 2011, he was a Visiting Professor at the EMC Group, Dipartimento di Elettronica, Politecnico di Torino, Italy. His current research interests comprise computational electromagnetics, electromagnetic compatibility, signal and power integrity, and antenna design. Dr. Vande Ginste was awarded the International Union of Radio Science (URSI) Young Scientist Award at the 2011 URSI General Assembly and Scientific Symposium. He is a Member of the IEEE. 